\title{
Valoración de la proporcionalidad de los pliegues cutáneos entre futbolistas profesionales titulares y reservas peruanos
}

\section{(Assessment of the proportionality of skin folds between professional footballers and alternate, Peruvian)}

\author{
M.A. COSSIO-BOLAÑOS ${ }^{1,5}$, J.E. HESPANHOL ${ }^{2}$, D. PORTELLA ${ }^{3}$, Y. MUNIZ DA SILVA ${ }^{3}$, \\ C. PABLOS ABELLA ${ }^{4}$, V. MASI ALVES ${ }^{5}$, R. VARGAS VITORIA ${ }^{1}$, M. ARRUDA ${ }^{3}$ \\ ${ }^{1}$ Departmento de Ciencias de la Actividad Física, Universidad Católica del Maule, Chile \\ ${ }^{2}$ Facultad de Educación Física, Pontificia Universidad Católica de Campinas, Campinas, Brasil \\ ${ }^{3}$ Facultad de Educación Física, Universidad Estadual de Campinas, Brasil \\ ${ }^{4}$ Instituto de Ciencias de la Actividad Física y Deporte de la Universidad Católica de Valencia, España \\ ${ }^{5}$ Instituto del Deporte Universitario, IDUNSA, Arequipa, Perú
}

\begin{abstract}
Resumen
El objetivo del estudio fue proporcionar información descriptiva sobre las diferencias de proporcionalidad de la masa corporal y pliegues cutáneos entre jugadores titulares y reservas en función de la posición de juego. Fueron seleccionados de forma no probabilística 4 clubes profesionales de Primera División del Fútbol Profesional Peruano, considerándose 44 titulares y 28 reservas. Se evaluó la masa corporal, estatura, seis pliegues cutáneos, determinándose la proporcionalidad corporal por medio de la estrategia del Phantom. Los resultados muestran que la proporcionalidad de la masa corporal y los pliegues cutáneos es similar, tanto de forma general y por ubicación de juego, no existiendo diferencias significativas entre las posiciones de juego y entre titulares y suplentes. En conclusión, todos los jugadores, independientemente de la posición de juego y la titularidad y/o suplencia muestran similares valores de proporcionalidad corporal y de pliegues cutáneos, mostrando en general altos valores de masa corporal y bajos niveles de tejido adiposo.
\end{abstract}

Palabras clave: Proporcionalidad, pliegues cutáneos, fútbol.

\begin{abstract}
The aim of the study was to provide descriptive information about the differences in proportionality of body mass and skinfold thickness between starters and reserves as playing position. They were selected in a non-probabilistic 04 professional clubs in the First Division of Professional Football Peruvian, considering 44 starters and 28 reserves. We assessed body mass, height, six skinfolds, body proportionality determined by the strategy of the Phantom. The results show that the proportionality of body mass and skinfold is similar, both generally and by playing position, with no significant differences between playing positions and titular or alternate players. In conclusion, all players regardless of playing position and title and/or substitution proportionality show similar values and skinfold body, showing generally high body mass values and low levels of fat.
\end{abstract}

Keywords: Proportionality, skinfolds, football.

Correspondencia:

Marco Antonio Cossio Bolaños

Universidad Católica del Maule. Departamento de Ciencias de la Actividad Física

Av. San Miguel 3605. Talca - Chile

E-mail: mcossio30@hotmail.com 


\section{Introducción}

La cineantropometría fue presentada por primera vez como una técnica en desarrollo en el congreso Internacional de Ciencias de la actividad Física realizado simultáneamente con los juegos Olímpicos de Montreal en 1976 [1] y posteriormente fue definida por Ross [2] como una ciencia que estudia la relación entre la estructura y función humana. En este sentido, el estudio morfológico del atleta por medio de la cineantropometría se ha orientado principalmente desde el análisis antropométrico por variable, por la composición corporal, el somatotipo y la proporcionalidad [3]. Estas áreas son ampliamente utilizadas y estudiadas en atletas con la intención de estandarizar el tamaño, forma, proporción, composición, maduración e inclusive relacionar las variables antropométricas con las respuestas adaptativas al entrenamiento y el rendimiento deportivo [4,5]. Desde esa perspectiva Ross y Marfell-Jones [6] consideran que la cuantificación de la constitución morfológica de los atletas puede conducir a una mejor relación entre la constitución y el funcionamiento y el desempeño del deportista durante su competencia, inclusive el perfil antropométrico de los atletas puede ser utilizado para la detección de talentos [7], para la comparación entre países [8] o para la comparación de la evolución de tendencias [9], entre otros aspectos. Es evidente entonces, que el tamaño, la estructura y las proporciones corporales, así como la composición corporal, son factores fundamentales que se encuentran relacionados directamente con el rendimiento deportivo [10], ya que a través del modelo humano unisexuado de similitud geométrica conocido como Phantom propuesto por Ross y Wilson [11] se puede generar una escala corporal uniforme y calcular el valor de la división de dos dimensiones antropométricas.

En consecuencia, varios son los estudios que consideran que el uso de la estrategia del Phantom permite verificar similitudes y diferencias entre atletas $[3,10,12,13]$, destacando de esta forma Ross y Marfell-Jones [6], que es útil para diferenciar grupos específicos, así como también Shepard y col [14] lo consideran relevante para la predicción inmediata del éxito en varios deportes y en la determinación de la madurez, respectivamente.

Respecto a estudios previos que engloban variables antropométricas en futbolistas profesionales peruanos, podemos destacar el estudio efectuado por Cossio Bolaños y Arruda [15] en el que proponen criterios de clasificación del \% de grasa corporal para futbolistas peruanos. A su vez es sorprendente destacar la ausencia de estudios en relación a la proporcionalidad de variables antropométricas por posiciones de juego, ya que en su mayoría, son varios los estudios internacionales que reportan las diferencias en función del tamaño y la composición corporal entre las posiciones de juego [16-18]. Por lo tanto, la hipótesis del estudio se fundamenta en que podría observarse diferencias entre los jugadores titulares y reservas en relación a la proporcionalidad de la masa corporal y los pliegues cutáneos cuando son comparados por posición de juego. De esta forma, el objetivo del presente estudio fue proporcionar información descriptiva sobre las diferencias de la proporcionalidad de la masa corporal y los pliegues cutáneos entre jugadores titulares y reservas en función de la posición de juego.

\section{Metodología}

Se realizó un estudio de tipo descriptivo-transversal. Las variables antropométricas fueron evaluadas al final del periodo competitivo de los años 2006, 2007 y 2008 (Diciembre) en las instalaciones del Instituto del Deporte Universitario de la Universidad Nacional de San Agustín de Arequipa, Perú. Los datos fueron recogidos en el periodo de la mañana (8:00-9:00am) en un laboratorio cerrado, manteniendo una temperatura de $22-24^{\circ} \mathrm{C}$.

Fueron seleccionados de forma no-probabilística (accidental) 4 clubes profesionales de la Liga de Primera División del Fútbol profesional peruano. Los jugadores desarrollaban un microciclo de 8-10 sesiones de entrenamiento por semana, teniendo cada sesión una duración aproximada de $90 \mathrm{~min} /$ día y el número de competiciones oficiales por año fue de 44 partidos, respectivamente.

\section{Sujetos}

Se evaluaron 72 futbolistas profesionales. Los jugadores fueron divididos en dos grupos: titulares y reservas. Para pertenecer al grupo de titulares, los jugadores debían haber completado al menos 38 partidos de 90 minutos cada uno durante la temporada anterior, lo que representa el $86 \%$ de los partidos disputados. En este grupo se consideró 44 jugadores de los cuatros clubes estudiados. En el caso de los jugadores denominados reservas, se consideró a los que habían completado un máximo de 6 partidos (14\%) de 90 minutos y/o fracción durante la última temporada.

Todos los jugadores a la fecha de la evaluación se encontraban en las mejores condiciones físicas, técnicas, tácticas y psicológicas. Posteriormente, 
los jugadores fueron clasificados en 4 posiciones de juego: Portero, defensas, medios y delanteros. Todos los jugadores firmaron la ficha de consentimiento para autorizar las evaluaciones antropométricas. El estudio también contó con la respectiva autorización del Comité de Ética del Instituto del Deporte Universitario de la Universidad Nacional de San Agustín, Arequipa, Perú.

\section{Técnicas y procedimientos}

Para la valoración de las medidas antropométricas se siguieron las normas y técnicas recomendadas por el International Working Group of Kineanthropometry descrita por Ross y MarfellJones [19]. Las variables medidas engloban la masa corporal, estatura y seis pliegues cutáneos. Tales variables fueron evaluadas por un experto antropometrista con certificación ISAK de Nivel 3. Las variables medidas muestran un Error Técnico de Medida (ETM) inferior al 3\% para las variables de masa corporal $(\mathrm{kg})$, estatura $(\mathrm{m})$, pliegue suprailíaco y abdominal y del $2 \%$ para los pliegues tricipital, subescapular, muslo y pantorrilla, respectivamente

- Masa corporal (kg): se utilizó una balanza digital con una precisión de $200 \mathrm{~g}$ de marca Tanita con una escala de 0 a $150 \mathrm{~kg}$.

- Estatura (cm): fue evaluada utilizando un estadiómetro de aluminio graduado en milímetros, de marca Seca, presentando una escala de 0-2,50 m.

- Pliegues cutáneos: Se evaluó la doble capa de piel de la región tricipital, subescapaular, supra-iliaco, abdominal, muslo y pantorrilla media utilizando un compás de pliegues cutáneos de Marca Harpenden con una presión constante de $10 \mathrm{~g} / \mathrm{mm} 2$.

Para la valoración de la proporcionalidad se utilizó la estrategia del Phantom, diseñado por Ross y Wilson [11] y revisado por Ross y Ward [20]. Los cálculos fueron realizados utilizando la fórmula:

$$
z=\frac{1}{S}\left[L .\left(\frac{170,18}{h}\right)^{d}-\text { Pphantom }\right]
$$

donde:

- Z: Índice Z-score de la variable estudiada.

- S: Desviación estándar del Phantom (rela tivo a la variable estudiada).

- L: variable evaluada del sujeto.

-170,18: constante de la estatura del phantom.

- h: estatura del sujeto estudiado.
- P: valor medio del Phantom.

- $\mathrm{d}$ : exponente unidimensional:

o 1 para longitudes, anchuras, perí metros y grosores de pliegues cutáneos

o 2 para todas las áreas

o 3 para las masas y volúmenes

Los resultados expresan valores numéricos de Z-score, cuyo significado implica el aumento o disminución de la variable antropométrica evaluada, donde el valor de $\mathrm{Z}$ puede ser positivo o negativo.

\section{Análisis estadístico}

Para verificar la distribución normal de la muestra se utilizó la prueba de normalidad de Kolmogorov-Smirnov. Para el análisis descriptivo se utilizó la media aritmética y desviación estándar. Para determinar las diferencias significativas intra-grupos (entre ubicaciones de juego) se utilizó ANOVA de dos vías $(\mathrm{p}<0,001)$ y para determinar las diferencias inter-grupos (titulares vs suplentes) se utilizó la prueba de ' $t$ ' de Student para muestras independientes $(p<0,001)$. Todo el análisis estadístico se llevó a cabo por medio del programa Sigma Estat 2.0.

\section{Resultados}

La tabla 1 muestra las características antropométricas de los jugadores titulares y suplentes en función de la posición de juego. Las variables consideradas son la edad, experiencia profesional, masa corporal, estatura y seis pliegues cutáneos. Los valores están representados en promedios y desviaciones estándar. Los resultados muestran que los porteros son más pesados y altos en relación a los medios, tanto en la serie de jugadores titulares, como de suplentes.

La proporcionalidad de la masa corporal y los pliegues cutáneos, tanto de los jugadores titulares y reservas en función de la posición de juego se puede observar en las figuras 1 y 2 . Los resultados muestran similitud en la proporcionalidad de la masa corporal y en los seis pliegues cutáneos. No se evidenció diferencias significativas en las 7 variables estudiadas. En cuanto a la masa corporal, los jugadores de las 4 posiciones de juego muestran valores positivos en relación al Phantom, a su vez, respecto a los pliegues cutáneos los valores muestran scores negativos, lo que explica un bajo nivel de distribución de tejido adiposo y una elevada masa corporal, tanto en jugadores titulares y reservas. 


\begin{tabular}{|c|c|c|c|c|}
\hline \multirow{2}{*}{ Variables } & \multicolumn{4}{|c|}{ Titulares } \\
\hline & Porteros $(n=4)$ & Defensas $(n=16)$ & $\operatorname{Medios}(n=16)$ & Delanteros $(\mathrm{n}=8)$ \\
\hline Edad (años) & $28,30 \pm 6,20$ & $30,20 \pm 4,90$ & $28,18 \pm 4,70^{*}$ & $30,12 \pm 5,84$ \\
\hline Experiencia profesional (años) & $9,50 \pm 4,70$ & $10,30 \pm 4,18$ & $8,81 \pm 4,60^{*}$ & $10,87 \pm 5,24$ \\
\hline Masa Corporal (kg) & $86,40 \pm 8,80$ & $78,24 \pm 8,40$ & $74,73 \pm 7,78^{\mathrm{a}}$ & $80,65 \pm 4,61$ \\
\hline Estatura (m) & $1,87 \pm 0,03$ & $1,79 \pm 0,06$ & $1,75 \pm 0,05^{\mathrm{a}}$ & $1,81 \pm 0,05$ \\
\hline \multicolumn{5}{|l|}{ Pliegues cutáneos (mm): } \\
\hline Tricipital & $7,80 \pm 0,93$ & $7,08 \pm 2,14$ & $7,88 \pm 2,44$ & $7,80 \pm 3,56$ \\
\hline Subescapular & $10,45 \pm 1,98$ & $10,87 \pm 2,47$ & $10,98 \pm 2,66$ & $11,35 \pm 2,30$ \\
\hline Supra-ilíaca & $12,25 \pm 3,11$ & $12,02 \pm 4,14$ & $15,02 \pm 7,02$ & $10,73 \pm 4,11$ \\
\hline Abdominal & $14,2 \pm 5,39$ & $14,82 \pm 5,04$ & $15,28 \pm 5,49$ & $12,23 \pm 4,80$ \\
\hline Muslo & $9,95 \pm 4,09$ & $8,82 \pm 3,04$ & $7,91 \pm 2,05$ & $8,75 \pm 3,10$ \\
\hline Pantorrilla & $5,85 \pm 1,08$ & $4,20 \pm 1,32$ & $4,66 \pm 1,28$ & $4,93 \pm 2,04$ \\
\hline \multirow[t]{3}{*}{$\sum 6$ pliegues cutáneos $(\mathrm{mm})$} & $60,50 \pm 11,90$ & $57,82 \pm 14,50$ & $61,75 \pm 16,93$ & $55,77 \pm 17,44$ \\
\hline & \multicolumn{4}{|c|}{ Reservas } \\
\hline & Porteros $(n=4)$ & Defensas $(n=8)$ & Medios (n=9) & Delanteros $(n=7)$ \\
\hline Edad (años) & $27,50 \pm 6,24$ & $25,11 \pm 2,93$ & $23,75 \pm 3,98$ & $26,71 \pm 4,60$ \\
\hline Experiencia profesional (años) & $9,50 \pm 4,43$ & $5,88 \pm 2,26$ & $4,75 \pm 2,86$ & $7,14 \pm 4,05$ \\
\hline Masa Corporal (kg) & $78,72 \pm 3,55$ & $74,77 \pm 6,84$ & $69,45 \pm 6,85^{\mathrm{a}}$ & $76,28 \pm 5,08$ \\
\hline Estatura (m) & $1,83 \pm 0,03$ & $1,77 \pm 0,05$ & $1,72 \pm 0,04^{\mathrm{a}}$ & $1,78 \pm 0,06$ \\
\hline \multicolumn{5}{|l|}{ Pliegues cutáneos (mm): } \\
\hline Tricipital & $7,30 \pm 1,03$ & $7,37 \pm 2,69$ & $6,90 \pm 2,40$ & $7,03 \pm 2,55$ \\
\hline Subescapular & $10,15 \pm 1,28$ & $11,08 \pm 2,56$ & $10,55 \pm 2,81$ & $10,31 \pm 1,11$ \\
\hline Supra-ilíaca & $1160 \pm 1,90$ & $10,55 \pm 2,84$ & $12,47 \pm 5,24$ & $9,74 \pm 3,32$ \\
\hline Abdominal & $12,95 \pm 4,05$ & $12,40 \pm 2,73$ & $13,75 \pm 7,35$ & $11,60 \pm 3,39$ \\
\hline Muslo & $10,75 \pm 3,71$ & $8,76 \pm 3,13$ & $7,59 \pm 1,97$ & $8,85 \pm 2,90$ \\
\hline Pantorrilla & $5.65 \pm 1,18$ & $5,22 \pm 2,60$ & $4,92 \pm 1,30$ & $4,72 \pm 1,60$ \\
\hline$\sum 6$ pliegues cutáneos (mm) & $58,40 \pm 10,06$ & $55,40 \pm 13,22$ & $56,17 \pm 16,63$ & $53,27 \pm 12,45$ \\
\hline
\end{tabular}

Leyenda: $\sum$ : sumatoria de pliegues, *: diferencias con relación a los suplentes, a: diferencia con relación a los porteros.

Tabla 1. Características generales de la muestra estudiada.

Las comparaciones de la proporcionalidad de la masa corporal y los pliegues cutáneos entre jugadores de la misma posición de juego (titulares vs suplentes) se observa en la figura 3. En las 4 posiciones de juego, tanto de jugadores titulares como de reservas se observó valores positivos de masa corporal y valores negativos en los pliegues cutáneos.

En relación a la comparación de los Z-scores de la masa corporal y los pliegues cutáneos, no se observó diferencias significativas entre los porteros, defensas, medios y delanteros titulares y reservas $(\mathrm{p}>0,001)$, Por lo tanto, la distribución de los pliegues cutáneos de los jugadores titulares de las cuatro posiciones de juego son similares a los jugadores suplentes de las mismas posiciones de juego, respectivamente. 


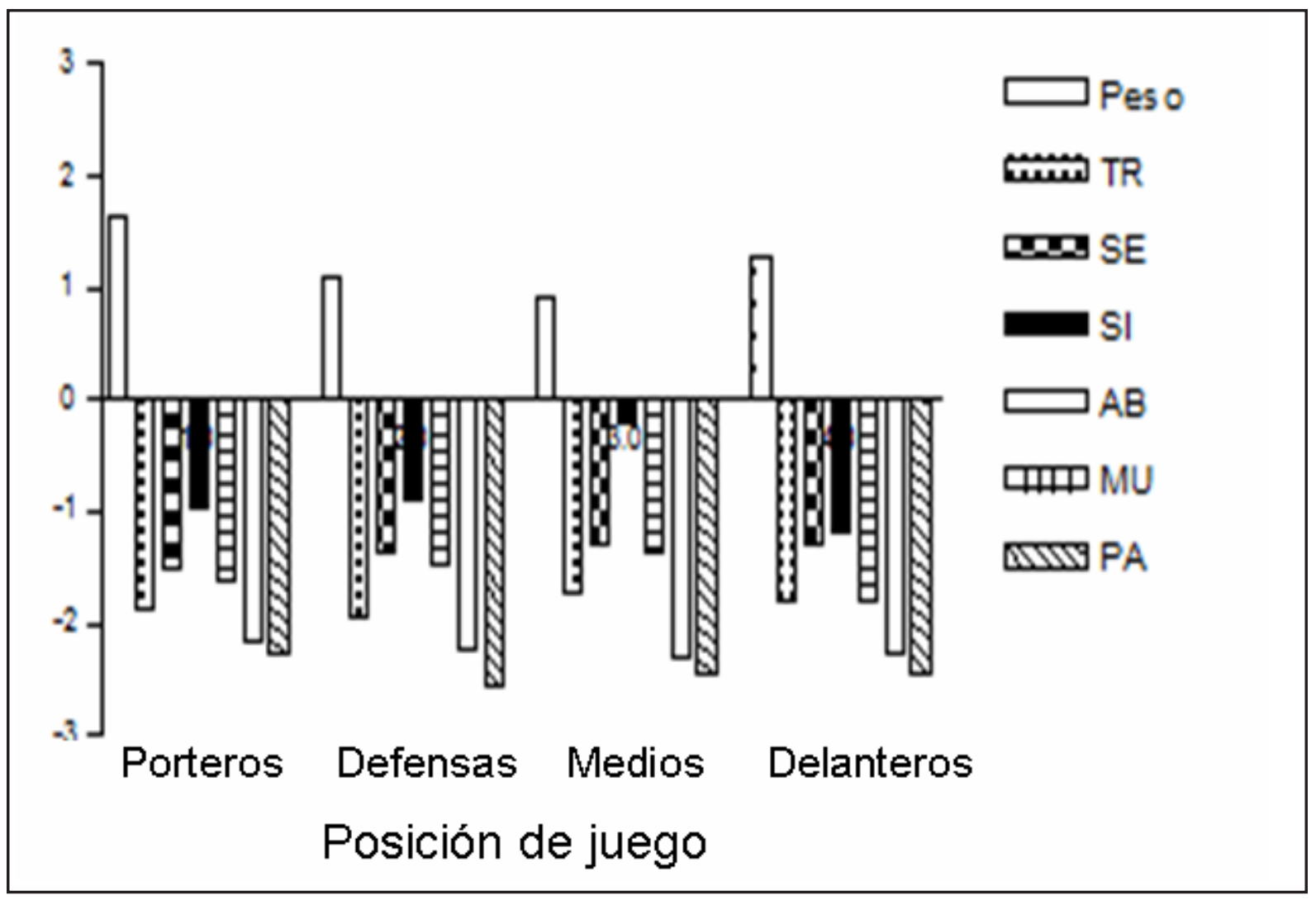

Figura 1. Representación gráfica del Z-score (Phantom) de la masa corporal y los pliegues cutáneos de futbolistas titulares en función de la posición de juego.

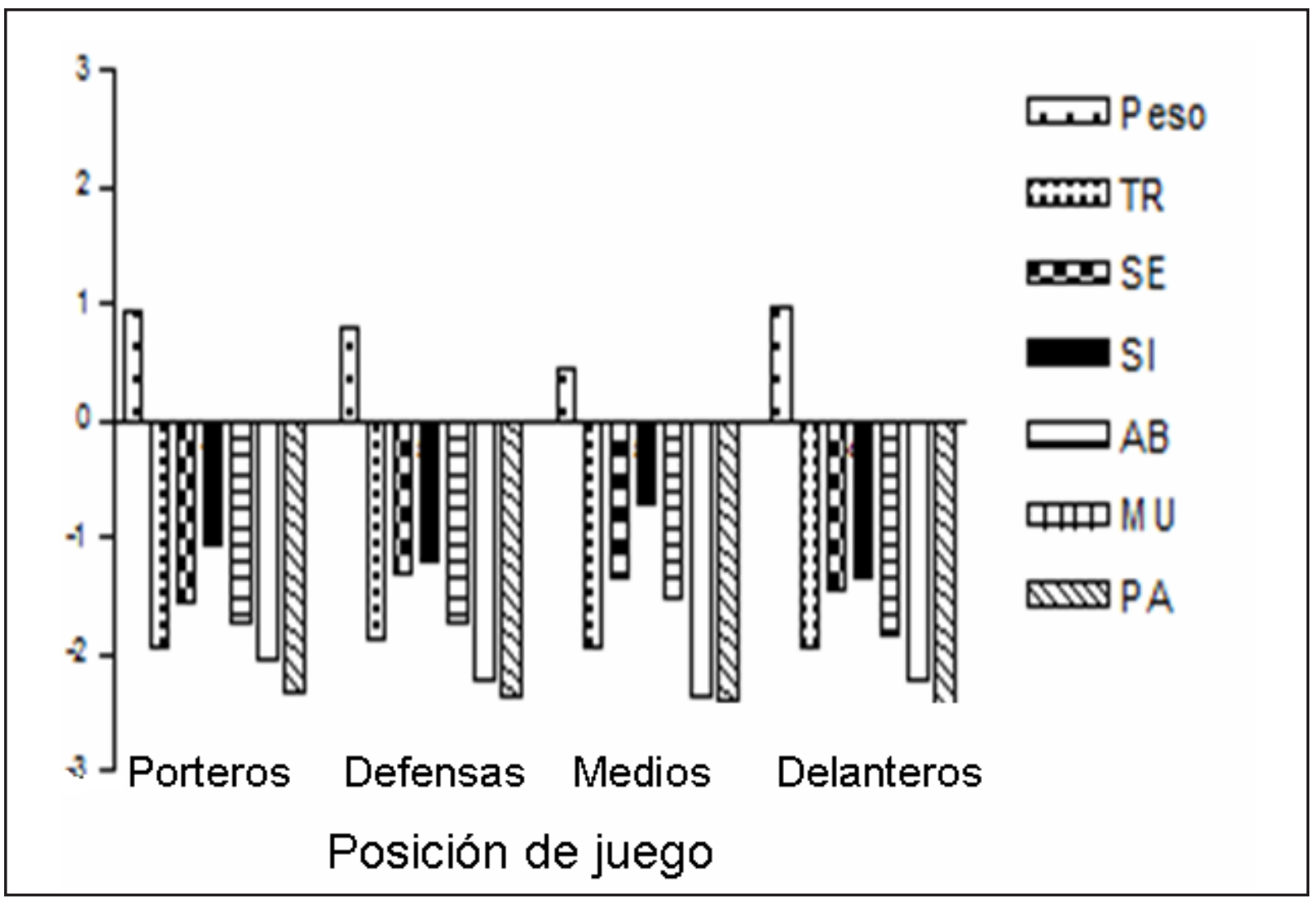

Figura 2. Representación gráfica del Z-score (Phantom) de la masa corporal y los pliegues cutáneos entre jugadores titulares y reservas de la misma posición de juego. 


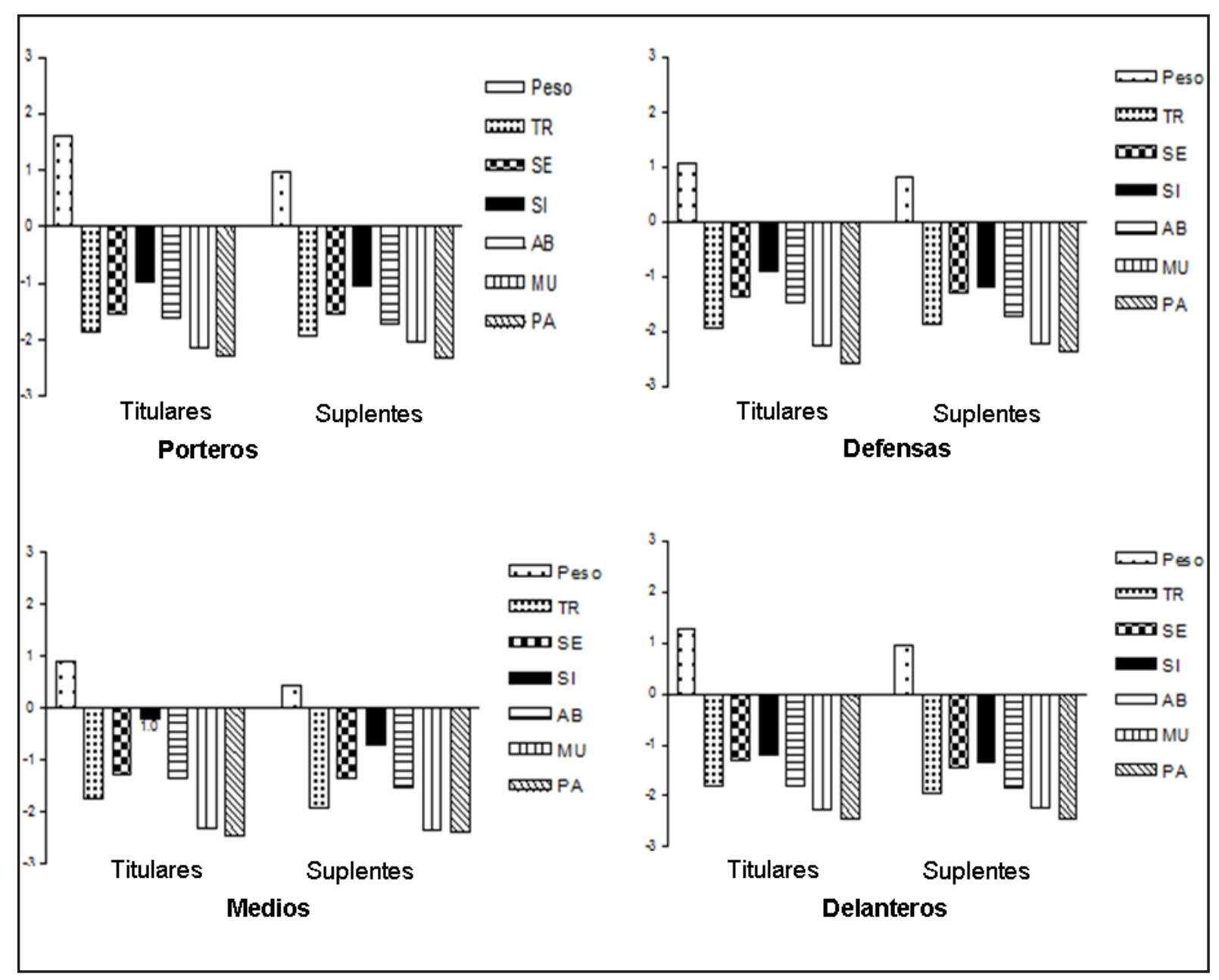

Figura 3. Representación gráfica del Z-score (Phantom) de la masa corporal y los pliegues cutáneos entre jugadores titulares y reservas de la misma posición de juego.

\section{Discusión}

Los resultados obtenidos en el presente estudio de forma general muestran que las variables antropométricas de masa corporal y estatura, son similares a estudios locales $[15,21]$ e internacionales $[16,18,22]$. Esto evidencia que existe poca diferencia entre los jugadores de élite de diversas realidades. Sin embargo, son varios los estudios que demostraron diferencias en cuanto al tamaño y composición corporal por ubicaciones de juego $[18,24]$ donde generalmente los porteros y defensas son los más altos y pesados en relación a las demás posiciones de juego, sin embargo, en el presente estudio se evidenció que los porteros son los más altos y pesados, y los medios son los más bajos y livianos.

Respecto a la edad, los titulares presentan valores relativamente superiores en relación a los reservas y presentan mayor experiencia profesional. En este sentido, se destaca que tanto los ju- gadores titulares y reservas se encuentran dentro del rango de edad que algunos estudios reportaron $[13,18,25]$, así como en cuanto a la experiencia profesional [26]. Estos resultados permiten destacar que las comisiones técnicas de los cuatro clubes estudiados parecen interesarse en convocar a los jugadores de mayor experiencia para que se desempeñen como titulares en la mayoría de los partidos en el fútbol Peruano.

En relación a la proporcionalidad de la masa corporal y los pliegues cutáneos de futbolistas titulares y reservas, en función de la posición de juego, los resultados mostraron Z-score de Phantom similares para las cuatro posiciones de juego, no evidenciándose diferencias en las ubicaciones de juego, por lo que, tanto porteros, defensas, medios y delanteros titulares y reservas muestran un mismo patrón de comportamiento en cuanto a la masa corporal y los pliegues cutáneos, respectivamente. Estos resultados también fueron observados en futbolistas sudamericanos de élite [13], en atletas 
mexicanos de varias modalidades deportivas (futbol, natación, remo y atletismo) [27], en atletas de élite de juegos olímpicos y campeonatos del mundo de acuerdo a una valoración estándar [28] y en futbolistas universitarios de salón de México [3], a pesar de presentar estos últimos mayor distribución de tejido adiposo en relación a los atletas profesionales de élite y del presente estudio.

De esta forma, en relación al Z-score del Phantom se observa valores positivos de masa corporal y valores negativos en los seis pliegues cutáneos. Por lo tanto, los futbolistas del estudio mostraron bajos niveles de adiposidad y altos valores de masa corporal. Desde esa perspectiva, en general, los atletas son más ligeros y presentan menor espesor en sus pliegues cutáneos [27], por lo que el porcentaje de grasa corporal a menudo en los futbolistas oscila entre 9-12\% [15] y valores superiores e inferiores podría afectar en el rendimiento deportivo de los mismos. Sin embargo, los valores positivos del peso corporal se explican por la mayor masa muscular producto de los años de entrenamiento, aunque en el estudio no fue posible la valoración de la composición corporal (masa muscular, grasa, ósea y residual), lo que hubiera permitido una mejor explicación de la proporcionalidad a través del fraccionamiento en compartimientos corporales de los futbolistas titulares y suplentes, respectivamente. Esto limita los resultados observados en el presente estudio, por lo que se recomienda analizar los resultados con precaución.

La valoración de la proporcionalidad entre jugadores titulares y reservas de la misma posición de juego, permitió determinar que tanto los porteros, defensas, medios y delanteros presentaron similares Z-score para los pliegues cutáneos y para la masa corporal. Estos resultados evidencian que desde el punto de vista de la proporcionalidad analizada mediante la estrategia Phantom no fue posible verificar diferencias entre jugadores titulares y suplentes de la misma posición de juego en futbolistas profesionales peruanos, a pesar de que varios autores sugieren su uso como modelo comparativo para diferenciar grupos [12], para la predicción inmediata del éxito en varios deportes [3] y para relacionar con el rendimiento deportivo [27] el cual puede ser considerado según Rivera Sosa [3] como un dispositivo de cálculo y no como un sistema normativo. En este sentido, se necesitan más investigaciones para estudiar la proporcionalidad corporal de futbolistas titulares y reservas, tal vez, considerando otros criterios de inclusión y variables antropométricas como circunferencias y diámetros óseos.
En consecuencia, los datos que se dispusieron para hacer el estudio fueron obtenidos básicamente de la lista que los entrenadores (comisiones técnicas) elaboraron para llevar el control del número de partidos jugados por jugador durante la competición en cada temporada. Este criterio podría ser una segunda limitación para los resultados del presente estudio, puesto que algunos jugadores, para determinados entrenadores podrían ser considerados como titulares y/o reservas. Por lo tanto, el grupo de futbolistas profesionales peruanos estudiados (titulares y reservas) en función de la posición de juego parecen presentar masa corporal y pliegues cutáneos homogéneos. Aunque existe la posibilidad de variar la proporcionalidad a lo largo y al final de una temporada competitiva, como especifica Silvestre y Cols [29], en relación a los componentes del somatotipo. En este sentido, en el presente estudio cabe resaltar que los jugadores fueron evaluados al final del periodo competitivo, en el que probablemente exista una pequeña disminución del rendimiento deportivo, aunque es necesario considerar para futuros estudios la monitorización de la proporcionalidad de forma longitudinal a lo largo de una temporada.

Se concluye que la proporcionalidad de la masa corporal y los pliegues cutáneos de futbolistas profesionales peruanos titulares y reservas es similar, tanto de forma general y por ubicación de juego. Todos los jugadores, independientemente de la ubicación de juego y la titularidad y/o suplencia muestran altos valores de masa corporal y bajos niveles de tejidos adiposo en los seis pliegues valorados. Los resultados sugieren valores similares de tejido adiposo en ambos grupos de estudio.

\section{Referencias}

1. Rose EH, Pigatto E, Rose RCF. Cineantropometria, educação física e treinamento desportivo. Rio de Janeiro: FAE, 1984, p.80.

2. Ross W. Kinanthropometry: an emerging science technology. In: Landry, F, and Orban, W. Biomechanics of sport and kinanthropometry. Miami, Symposia specialists, 1978.

3. Rivera Sosa JM. Valoración del somatotipo y proporcionalidad de futbolistas universitarios mexicanos respecto a futbolistas profesionales. Revista Internacional de Medicina y Ciencias de la Actividad Física y el Deporte 2006;6 (21):1628.

4. Carter JEL, Heath BH. Somatotiping - develpment and aplications. London: Cambridge University Press, 1990.

5. Al-Hazzaa HM, Almuzaini KS, Al-Refaee SA, Sulaiman MA, Dafterdar MY, Al-Ghamedi 
A, Al-Khuraiji KN. Aerobic and anaerobic power characteristics of Saudi elite soccer players. J Sports Medicine and Physical Fitness 2001;41(1):54-8.

6. Ross WD, Marfell-Jones MJ, En MacDougall JD, Wenger HA, Green HJ (Eds.). Evaluación fisiológica del deportista. Ed. Paidotribo: Barcelona, 2000, p. 277-379.

7. Franks AM, Williams AM, Reilly T, Nevill A. Talent identification in elite youth soccer players: Physical and physiological characteristics. Communication to the 4th World Congress on Science and Football, Sydney. J Sports Sci 1999;17:812.

8. Doutreloux JP, Artigot A, Thon B. Etude de la morphologie des joueurs de rugby en fonction du poste et du niveau de compétition. Sciences et Techniques des Activités Physiques et Sportives 2000;52:7-20.

9. Olds $\mathbf{T}$. The rise and fall of anthropometry. J Sports Sci 2004;22(4):319-20.

10. Padilla PJ, Taylor AW, Yuhasz MS, Velázquez MA. Características antropométricas de atletas mexicanos. Rev Med Hosp Gen Mex 2004;67(1):11-21.

11. Ross WD, Wilson NC. A stratagem for proportional growth assessment. Acta Paediatrica Belgica 1974;Suppl.28:169-82.

12. Ackland TR, Schreiner AB, Kerr DA. Absolute size and proportionality characteristics of World Championship female basketball players. J Sports Sci 1997; 15:485-90.

13. Rienzi E, Mazza JC, Carter JEL, Reilly T. Futbolista sudamericano de elite: Morfología, análisis del juego y performance. Rosario, Biosystem Servicio educativo, 1998.

14. Shephard RJ, Labarre R, Jéquier JC, Lavallee H, Rajic M, Volle M. The "Unisex Phantom," Sexual Dimorphism, and Proportional Growth Assessment. Am J Physl Anthropol 1985;67:40312.

15. Cossio-Bolaños MA, Arruda M. Propuesta de valores normativos para la clasificación de variables antropométricas en futbolistas profesionales. Biomecánica 2010;18(1):19-23.

16. Brewer J, Davis JA. A physiological comparison of English professional and semi-professional soccer players [Abstract]. In: Proceedings of the 2nd World Congress on Science and Football, Eindhoven, 1991.

17. Arnason A, Sigurdsson A, Gudmundsson I, Holme L, Engebretsen L, Bahr R. Physical fitness, injuries and team performance in soccer. Medicine and Science in Sports and Exercise 2004;36:278-85.
18. Sporis G, Jukic I, Ostojic SM, Milanovic D. Fitness profiling in soccer: physical and physiologic characteristics of elite players. J Strength Cond Res 2009;23(7):1947-53

19. Ross WD, Marfell-Jones MJ. Kinanthropometry. In: MacDougall JD, Wenger HA, Green HJ (Eds). Physiological testing of elite athlete. London, Human Kinetics, 1991, p. 223-308.

20. Ross WD, Ward R. Sexual dimorphism and human proportionality. In: Hall R, Sexual dimorphism in Homo sapiens. Praeger. New Cork, 1982.

21. Arruda M. Cossio-Bolanos MAC, Portella D. Los pliegues cutáneos como predictores del porcentaje graso en futbolistas profesionales. Biomecánica 2009;17(2):7-14.

22. Prado WL, Botero JP, Fernandes Guerra RL, Rodrigues CP, Cuvello LC, Damaso AR. Perfil antropométrico e ingestão de macronutrientes em atletas profissionais brasileiros de futebol, de acordo com suas posições. Rev Bras Med Esporte 2006;12(2).

23. Matković BR, Misigoj-Duraković M, Matković B, Janković S, Ruzić L, Leko G, Kondric M. Morphological differences of elite Croatian soccer players according to the team position. Coll Antropol 2003;27(Suppl 1):167-74.

24. Sutton L, Scott M, Wallace J, Reilly T. Body composition of English Premier League soccer players: Influence of playing position, international status, and ethnicity, J Sports Sci 2009;27(10):1019-26.

25. Rodríguez $\mathbf{C}$, Echegoyen $\mathbf{S}$. Características antropométricas y fisiológicas de jugadores de fútbol de la selección mexicana. Archivos de Medicina del Deporte 2005;22(105):33-7.

26. Mirkov DM, Nedeljkovic A, Kukolj M, Ugarkovic D, Jaric S. Evaluation of reliability of soccer-specific field tests. J Strength Cond Res 2008;22:1046-50.

27. Padilla Alvarado JR. Perfil de proporcionalidad y la velocidad del lanzamiento en jugadores de béisbol. Revista Internacional de Medicina y Ciencias de la Actividad Física y el Deporte 2010;10(37):93-116.

28. Kerr D, Ackland T, Schreiner A. The elite athlete assessing body shape, size, proportion and composition. Asia Pacific Journal Clinical Nutrition 1995;4:25-9

29. Silvestre R, Kraemer WJ, West C, Judelson DA, Spiering BA, Vingren JL, Hatfield DL, Anderson JM, Maresh CM. Body composition and physical performance during a National Collegiate Athletic Association Division I men's soccer season. J Strength Cond Res 2006:962-970. 